\title{
DESCRIPTION ET CYCLE BIOLOGIQUE D'HEMOLIVIA STELLATA N. G., N. SP., HÉMOGRÉGARINE DE CRAPAUDS BRÉSILIENS
}

\author{
G. PETIT*, I. LANDAU*, D. BACCAM*, R. LAINSON** \\ Collaboration technique: F. GONNET
}

RÉSUMÉ

Une nouvelle hémogrégarine, Hemolivia stellata n. g., n. sp. est décrite chez le crapaud Bufo marinus L. et le vecteur naturel, la tique Amblyomma rotondatum K. Elle présente de nombreux caractères originaux : - une schizogonie et des kystes à la fois dans les hématies et les cellules du système réticulo-endothélial; - une sporogonie entièrement dans les cellules digestives de la tique, avec un oocyste en forme d'étoile et des sporokinètes qui sortent de l'oocyste pour se diviser et donner des sporocystes chez le même individu hôte. Expérimentalement, les kystes et les sporocystes déterminent une infection parasitaire complète chez les crapauds sains qui les ingèrent.

Tous les modes d'infestation connus chez les hémogrégarines peuvent potentiellement être utilisés : ingestion d'un vecteur contenant des sporocystes; ingestion de sporocystes disséminés dans le milieu extérieur; prédation d'un hôte invertébré paraténique ayant ingéré des sporocystes du milieu extérieur; prédation d'un vertébré présentant des kystes d'attente dans les tissus.

Le genre le plus proche d'Hemolivia est Karyolysus; tous les deux présentent chez le vecteur deux phases de multiplication l'une donnant naissance aux sporokinètes, l'autre aux sporozoïtes.

Dans les deux genres la formation des sporokinètes s'effectue chez le vecteur qui s'est contaminé au cours du repas de sang; les sporocystes par contre se forment chez le même individu hôte pour Hemolivia et chez la descendance pour Karyolysus.

\section{Summary: Description and life cycle of Hemolivia stellata n. g., n. sp., a haemogregarine of brazilian toads.}

A new haemogregarine, Hemolivia stellata n. g., n. sp., is described in its natural hosts: the toad Bufo marinus and the vector tick, Amblyomma rotondatum. It shows many peculiar characteristics: - schizogony and cyst formation in the erythrocytes, as well as in reticulo endothelial cells, - sporogony developing inside the gut cells of the tick, with formation of a star-shaped oocyst and sporokinetes migrating from the oocyst to new digestive cells in the same host, where they divide and give rise to sporocysts containing sporozoites. Experimental transmission to clean toads was achieved by ingestion of sporocysts or cysts.

All modes of transmission known for haemogregarines are poten- tially active: ingestion of ticks with sporocysts, ingestion of sporocysts disseminated in the external environment, ingestion of an invertebrate paratenic host contaminated with sporocysts from the external environment, predation of a vertebrate host with tissue cysts.

The genus most closely related to Hemolivia is Karyolysus; both parasites undergo in the vector two successive multiplications, one giving rise to sporokinetes, the other to sporozoites. In contrast to Karyolysus, in which sporokinetes are formed in the adult mite and sporozoites in the offspring, in Hemolivia, sporokinetes and sporozoites are formed in the same individual tick.

\section{INTRODUCTION}

Les Bufo marinus de la région de Belém sont parasités par une hémogrégarine nouvelle dont le vecteur est une tique Amblyomma rotondatum (Petit et coll., 1987). La morphologie, le cycle biologique et la transmission expérimentale de ce parasite sont décrits dans cet article.

* Laboratoire de Zoologie-Vers, associé au CNRS, et Laboratoire de Protozoologie et Parasitologie comparée, EPHE, Muséum National d'Histoire Naturelle, F 75231 Paris Cedex 05.

** Wellcome Parasitology Unit, Instituto Evandro Chagas, Av. Almirante Barroso, 492, C. P. 621, 66.000 Belém, Para, Brésil.

Accepté le : 8 décembre 1989.

\section{MATÉRIELS ET MÉTHODES}

1 - Crapauds

a - Crapauds naturellement parasités

Ce sont des B. marinus capturés dans la région de Belém (État du Pará) au Brésil.

Une partie du matériel a été préparée sur place (frottis sanguins, autopsie), $4 \mathrm{~B}$. marinus ont été ramenés vivants à Paris pour l'étude de la transmission du parasite.

b - Crapauds non parasités

Seize crapauds de diverses provenances ont été utilisés à Paris pour l'étude de la transmission expérimentale du parasite et l'élevage de la tique vectrice; ce sont : 
- 3 B. marinus de Belém qui n'ont pas montré d'infection sanguine par Hémogrégarine, au cours des 6 mois qui ont suivi leur arrivée au laboratoire. Ces crapauds sont par ailleurs parasités par Dactylosoma sp. et par des sporozoïtes d'un Lankesterellidae. - -5 B. marinus de Guadeloupe sains.

- 8 B. poepigii du Pérou parasités par Lankesterella sp.

Tous ces batraciens sont maintenus en survie en terrarium où ils se nourrissent de souriceaux et de souris.

\section{2 - Tiques}

La sporogonie de cette hémogrégarine est étudiée chez $A$. rotondatum, une espèce parthénogénétique trouvée naturellement sur les $B$. marinus de Belém. Ces tiques sont abondantes; suivant les captures (effectuées entre mai et octobre), 30 à $100 \%$ des crapauds étaient parasités par différents stades de l'arthropode.

Certaines expériences ont été faites au Brésil, la plupart de l'étude a été réalisée à Paris avec l'élevage obtenu à partir d'une seule femelle adulte récoltée gorgée à Belem.

L'élevage de la descendance de cette tique se fait à $28^{\circ} \mathrm{C}$ dans une pièce à 80-90\% d'humidité.

\section{a - Cycle biologique de la tique} dans nos conditions d'élevage

- La femelle adulte pond des œufs entre le $5^{\mathrm{e}}$ et le $20^{\mathrm{e}}$ jour qui suivent le gorgement.

- Les larves hexapodes sortent des œufs au bout de 40 jours et peuvent se gorger de sang 8 jours plus tard. Elles muent et se transforment en nymphes 15 jours plus tard.

- Les nymphes peuvent faire un repas sanguin 8 à 10 jours après cette première mue. Elles muent 15 jours après le gorgement.

- Le jeune adulte formé après cette mue est capable de se gorger au bout de 8 jours.

Les temps indiqués ici ne tiennent pas compte de la durée du repas sanguin qui varie beaucoup suivant le stade de la tique, l'expérience, et les individus.

\section{b - Repas sanguin des tiques}

Les stades à jeun de l'arthropode (larve hexapode, nymphe ou adulte) sont placés dans un sac en tissu au contact du crapaud (sain ou parasité). Après $24 \mathrm{~h}$, les tiques sont fixées. Les crapauds sont alors placés sur une grille au-dessus d'un bac rempli d'eau ce qui permet de récolter facilement les stades gorgés.

- Durée du repas de la larve hexapode : entre 6 et 26 jours.

- Durée du repas de la nymphe : entre 7 et 23 jours.

- Durée du repas du sang de l'adulte : entre 14 et 39 jours.

Le repas sanguin se fait d'abord de façon lente et la majorité du sang est ingérée au cours du dernier tiers du temps de gorgement indiqué ci-dessus.

Après le repas de sang, les tiques récoltées sont placées dans des tubes en verre dont le fond renferme du papier filtre. Les tubes sont fermés par un coton cardé et placé dans des chambres humides constituées par un bocal en verre.

Le bocal n'est pas fermé hermétiquement et contient du coton humide à la base.

\section{3 - TeChNiques D'Étude du parasite}

Chez le crapaud, l'infection sanguine est étudiée sur des frottis de sang colorés au Giemsa après fixation au méthanol.

Le sang est prélevé au doigt du crapaud; le doigt est coupé à son extrémité et est frotté directement sur la lame (méthode qui nous a été indiquée par le $\mathrm{D}^{\mathrm{r}} \mathrm{J}$. R. Barta de l'Université de Toronto).

Les autopsies comprennent des frottis par appositions des différents organes et des fixations au Carnoy pour l'étude histologique (coloration au Giemsa).

L'infection chez la tique est étudiée soit à frais, après dilacération entre lame et lamelle dans du milieu physiologique, soit après coloration au Giemsa de coupes histologiques ou de dilacérations à sec sur lame.

\section{4 - Transmission expérimentale du Parasite (tableau I) \\ a -- Ingestion par les B. marinus de Guadeloupe de nymphes contenant le stade sporokinète du parasite}

Un lot de larves hexapodes est gorgé sur un crapaud bien parasité. Au bout de 15 jours, peu après la mue de la tique, les jeunes nymphes qui renferment de nombreux oocystes mûrs sont données à ingérer aux Bufo sains $1009 \mathrm{ET}$ et $1010 \mathrm{ET}$.

Les tiques sont placées vivantes dans la bouche des crapauds qui ingèrent chacun 60 nymphes, soit plusieurs milliers de sporokinètes.

b - Ingestion par le crapaud de nymphes contenant le stade sporocyste du parasite

\section{Expérience 1:}

Des nymphes du même lot que dans l'expérience précédente sont utilisées, mais cette fois, elles sont âgées de 25 jours et renferment des sporocystes.

Deux B. marinus sains (1007 et 1008 ET), provenant de Guadeloupe, sont infectés en utilisant la même méthode que précédemment, avec 50 nymphes chacun, soit plusieurs milliers de sporocystes.

\section{Expérience 2:}

Deux B. poepigii (37-38 ES) du Pérou ingèrent 60 nymphes âgées de 46 jours, gorgées sur un $B$. marinus de Belém infecté expérimentalement au laboratoire (909 ET).

\section{Expérience 3:}

Deux B. poepigii (39-40 ES) ingèrent 20 nymphes, âgées de 35 jours, gorgées sur un $B$. marinus de Belém infecté expérimentalement au laboratoire (868 ET).

\section{Expérience 4:}

Un B. marinus de Belém (909 ET) ne présentant pas de parasitémie sanguine depuis 5 mois est infecté par ingestion de 17 nymphes âgées d'environ 30 jours gorgées sur des crapauds naturellement infectés (893 ET et $889 \mathrm{ET}$ ).

\section{Expérience 5:}

Un B. marinus de Belém (868 ET) s'est infecté accidentellement en ingérant une nymphe parasitée qui venait de se gorger sur lui. Cette nymphe s'était infectée au stade larve hexapode ( $40 \mathrm{j}$ avant) par gorgement sur le crapaud 868 ET (infecté naturellement).

c - Ingestion par le crapaud

du foie d'un crapaud parasité contenant des kystes

Un B. poepigii du Pérou ( $588 \mathrm{XP}$ ) a reçu directement dans la bouche une partie du foie d'un crapaud naturellement parasité (877 ET).

Des appositions colorées du foie du Bufo donneur montrent 
TABLEAU I. - H. stellata dans les infections naturelles et expérimentales des Bufo. Proportion relative des différentes formes du parasite dans les hématies du doigt et les cellules réticuloendothéliales des organes internes.

\begin{tabular}{|c|c|c|c|c|c|c|}
\hline CRAPAUD ORIGIDE & $\begin{array}{l}\text { ThFES-A } \\
\text { TATION }\end{array}$ & गTOPSI & $\begin{array}{l}\text { HEMATIE } \\
\text { DU DOIGT }\end{array}$ & $\begin{array}{l}\text { HEMATIE } \\
\text { TISSUS }\end{array}$ & $\begin{array}{l}\text { SRE NON } \\
\text { PIGMEMTE }\end{array}$ & $\begin{array}{c}\text { SRE } \\
\text { PIGMEMTE } \\
\end{array}$ \\
\hline $\begin{array}{l}\text { B. marinus Belem } \\
\text { 49ET }\end{array}$ & Nat & IJ & Gom+ & $\begin{array}{l}\mathrm{K}+++ \\
\mathrm{SCn++} \\
\text { Gam++ }\end{array}$ & & \\
\hline $\begin{array}{l}\text { D. Marinus Belem } \\
\text { B77ET }\end{array}$ & Nat & $150 \mathrm{~s}$ & Gomt+t+ & $\begin{array}{l}\text { Scht+ } \\
\text { Gem+t+ }\end{array}$ & $\begin{array}{l}\text { K++++ } \\
\text { Sch++++ } \\
\text { Gom++ } \\
\end{array}$ & $\begin{array}{l}\mathrm{K+++} \\
\text { Gam++ }\end{array}$ \\
\hline $\begin{array}{l}\text { 9. marlinus Bélem } \\
\text { B6BET }\end{array}$ & Spcy & $300 \mathrm{~s}$ & Gom+t+ & $\begin{array}{l}\mathrm{K}+++ \\
\text { Sch++t++ } \\
\text { Gam++ }\end{array}$ & $\begin{array}{l}\text { Scht } \\
\text { Gom+ }\end{array}$ & $\begin{array}{l}+++ \\
\text { Gom+t++ }\end{array}$ \\
\hline $\begin{array}{l}\text { 9. marinus Bélem } \\
\text { 909ET }\end{array}$ & Spcy & $210 \mathrm{~d}$ & Gom+ & $\begin{array}{l}\text { Scht } \\
\text { Gom+ }\end{array}$ & Gom+t & $\begin{array}{l}\mathrm{K}++ \\
\text { Gom+t++ }\end{array}$ \\
\hline $\begin{array}{l}\text { D. marinus Guedeloupe } \\
\text { 1007ET }\end{array}$ & Spcy & NF & Merot & & & \\
\hline $\begin{array}{l}\text { 8. merinus Guadeloupe } \\
\text { lo0BET }\end{array}$ & SDCy & 1201 & Merot & $\begin{array}{l}\text { Scht } \\
\text { Mérot }\end{array}$ & $\begin{array}{l}\text { Scht } \\
\text { Merot }\end{array}$ & $k+$ \\
\hline $\begin{array}{l}\text { 8. marinus Guadeloupe } \\
\text { 1009ET }\end{array}$ & spk & $150 \mathrm{~s}$ & & & & \\
\hline $\begin{array}{l}\text { D. marinus Guedeloupe } \\
\text { lo10ET }\end{array}$ & Spk & $150 \mathrm{~s}$ & & & & \\
\hline $\begin{array}{c}\text { D.poepigil Perou } \\
\text { 37ES }\end{array}$ & Spcy & W & & & & \\
\hline $\begin{array}{c}\text { 0.poepigil Perou } \\
\text { 3oEs }\end{array}$ & SpCy & $81 \mathrm{~J}$ & & & $\begin{array}{l}\mathrm{K}+ \\
\text { Scht } \\
\text { Gom+ }\end{array}$ & \\
\hline $\begin{array}{c}\text { D.poeplgil Perou } \\
\text { 39ES }\end{array}$ & Spcy & 62J & Gam+ & sent & $\begin{array}{l}K+ \\
\text { Scht } \\
\text { Gemt }\end{array}$ & \\
\hline $\begin{array}{l}\text { B.poeplgil Perou } \\
\text { 4OES }\end{array}$ & SpCy & 69J & Gam+ & $\operatorname{sen}+$ & $\begin{array}{l}+ \\
\text { Scht } \\
\text { Gem* }\end{array}$ & \\
\hline $\begin{array}{l}\text { B.poepigil Parou } \\
\text { 5oexp }\end{array}$ & Kystes & 2100 & Gom+ & $\begin{array}{l}\text { Scht } \\
\text { Gom }\end{array}$ & $\begin{array}{l}\text { Scht } \\
\text { Gem+ }\end{array}$ & $k^{+}$ \\
\hline
\end{tabular}

$(++++)=$ stade très abondant, $( \pm)=$ stade très rare, $(\mathrm{Gam})$ $=$ gamétocyte, $(\mathrm{K})=$ kystes, $($ Méro $)=$ mérozoïte, $($ Nat $)=$ Infestation Naturelle, $($ Spcy) $=$ sporocystes, $($ Spk $)=$ sporokinètes, $(\mathrm{Sch})$ $=$ schizonte, $(\mathrm{SRE})=$ cellule du système réticulo-endothélial, $(\mathrm{NF})$ $=$ autopsie non pratiquée.

qu'il contient de nombreux kystes à l'intérieur des cellules réticuloendothéliales.

\section{$\mathrm{d}$ - Ingestion des sporocystes, par différents arthropodes}

Plusieurs hôtes expérimentaux ont été testés :

- des larves de diptères (Aedes aegypti, Culex pipiens, Culicoides nubeculosus, Anopheles stephensi),
- des larves de crustacé (azelles),

- des larves et adultes de criquet (Locusta migratoria).

Les larves aquatiques de diptères ou de crustacés ingèrent spontanément les sporocystes placés dans l'eau à leur contact.

Les larves de criquet sont gavés avec une pâtée d'herbe contenant les sporocystes.

Nous ne détaillerons pas par la suite les résultats de cette expérimentation, car chez tous ces invertébrés, les sporocystes ne font que transiter dans le tube digestif sans être altérés ni modifiés.

\section{ÉVOLUTION DE L'INFECTION CHEZ LA TIQUE}

La sporogonie se déroule entièrement dans le tube digestif de la tique; son déroulement est étroitement lié à l'activité digestive de l'arthropode.

\section{1 - Évolution des Cellules digestives APRÈS LE REPAS SANGUIN}

Trois types de cellules digestives interviennent directement dans le cycle; en accord avec les observations sur la digestion chez Rhipicephalus appendiculatus (Walker et coll., 1987) et Boophilus microplus (Agbede et coll., 1985); nous distinguons :

- les cellules sessiles (sessil cells) qui s'hypertrophient considérablement après le repas sanguin. Au bout de 15 jours, ces cellules dégénèrent et sont progressivement éliminées dans les fèces;

- les cellules phagocytaires (motil cells) qui ont une forte activité à la suite du repas sanguin et sont ensuite éliminées vers le $15^{\mathrm{e}}$ jour;

- les cellules souches (stem cells) qui évoluent pour remplacer les deux types cellulaires précédents lorsque ceux-ci disparaissent à la fin de la digestion du sang. Ce processus de régénération n'existe pas après le gorgement de l'adulte.

\section{2 - Évolution du parasite (fig. 1, 2 et 3)}

Après l'ingestion du sang par la tique, certains gamétocytes sortent de leur coque puis pénètrent individuellement dans les cellules « sessiles».

Quelle que que soit la durée du repas sanguin et le stade de la tique, la conjugaison et la fécondation ont lieu environ au moment où la tique gorgée se détache du crapaud (temps $\mathrm{J} 0)$.

L'oocyste formé passe ensuite par différents stades : stade sphérique à 1 noyau à $\mathrm{J} 1$, stade en étoile à un noyau à $\mathrm{J} 3$, stade mûr à sporokinètes différenciés à $\mathrm{J} 15$.

Après le $15^{\mathrm{e}}$ jour, les sporokinètes sortent de l'oocyste et pénètrent dans de nouvelles cellules digestives qui sont soit les grandes cellules digestives (sessil cell), soit les petites cellules digestives indifférenciées (stem cell) qui se mettent en place en vue d'un prochain repas sanguin.

La formation des sporocystes est rapide; plusieurs sporokinètes peuvent se développer dans une même cellule 

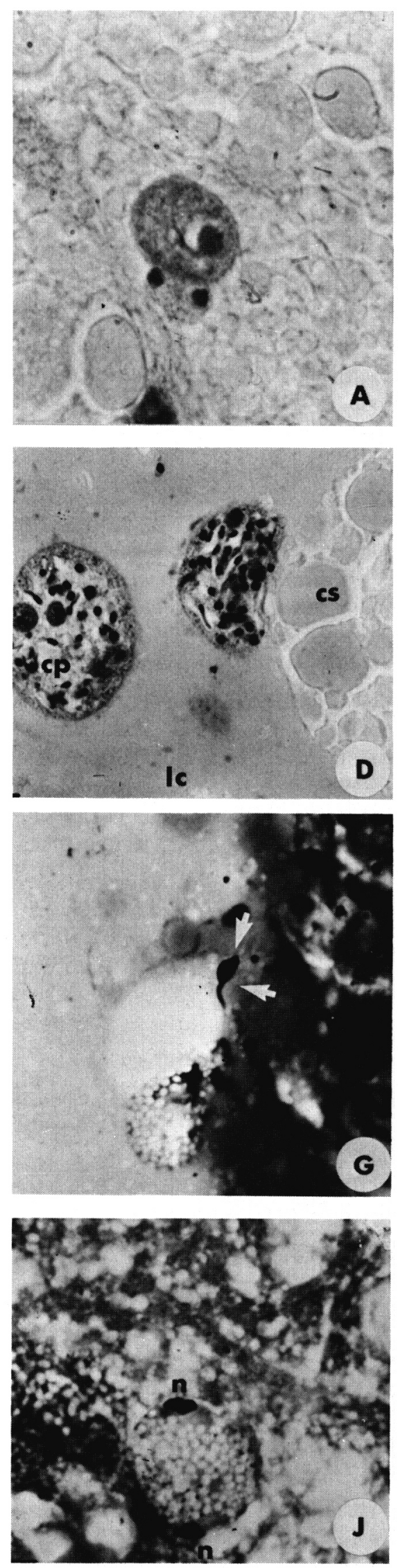
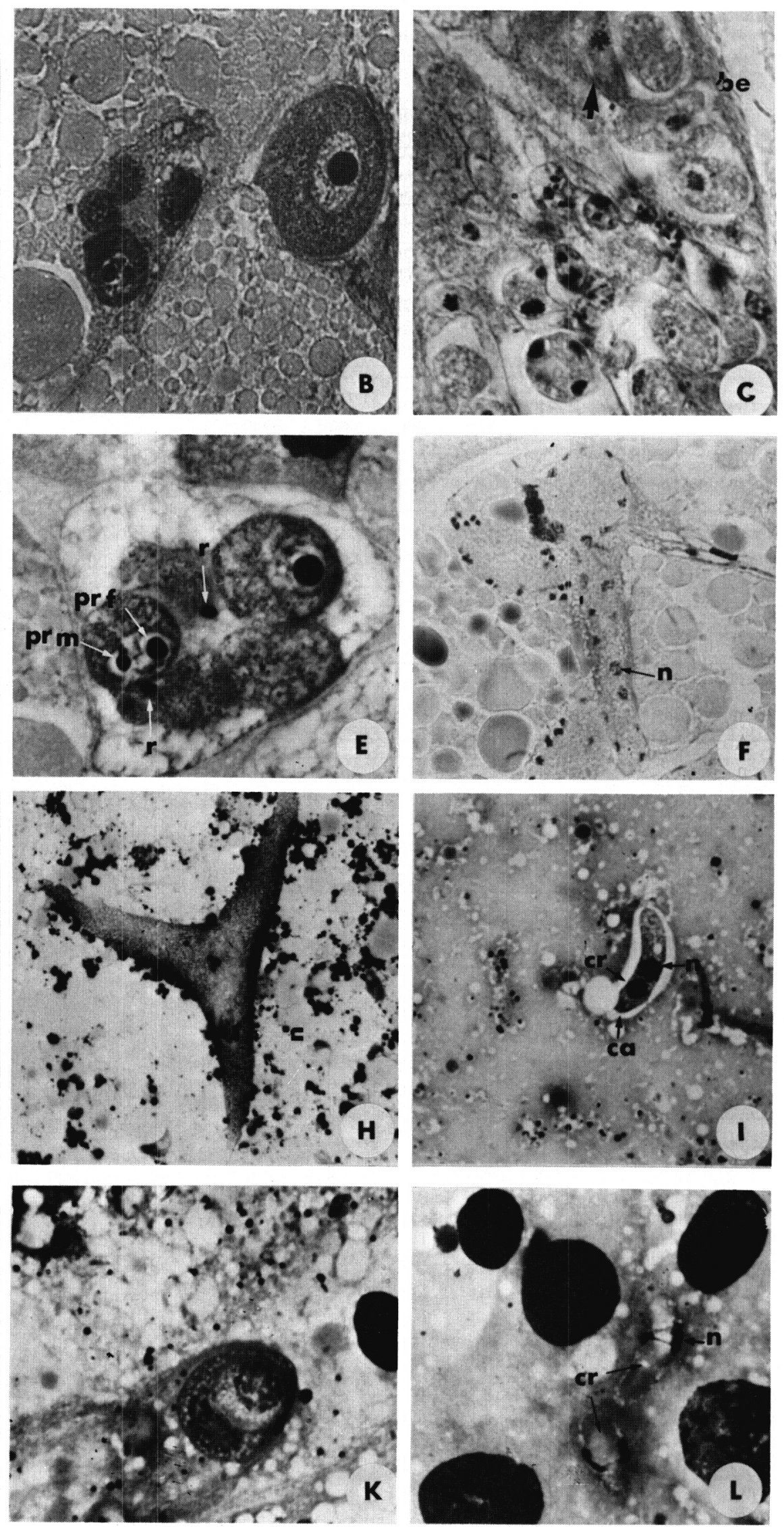
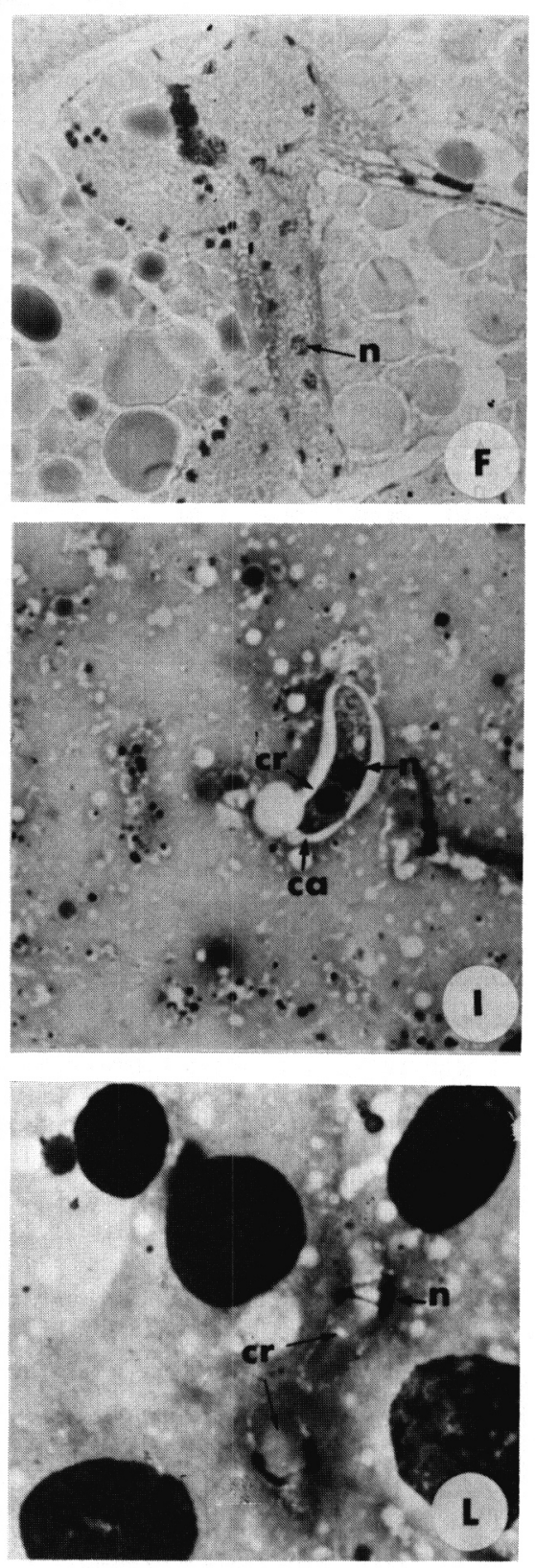
digestive. Étant donné le grand nombre de sporokinètes, le parasitisme du tube digestif devient alors souvent intense.

Chez la nymphe où la larve nous assistons au développement complet du cycle, chez l'adulte, le stade sporokinète n'est pas dépassé. Il correspond à la fin de la ponte et précède de peu la mort de la tique.

Il est possible d'observer des sporocystes chez l'adulte mais il s'agit alors de stades formés chez la nymphe et qui persistent après le gorgement de l'adulte.

Une partie des gamétocytes n'évolue pas, ils restent dans leur coque et sont absorbés par les cellules phagocytaires; ils sont ensuite digérés plus ou moins rapidement. Certains peuvent résister pendant une quinzaine de jours, jusqu'à ce que ces cellules dégénèrent et soient éliminées dans les fèces.

\section{3 - DeVEniR DES SPOROCYSTES}

\section{a - Sporocystes formés dans les cellules souches}

Les sporocystes formés dans les cellules souches (stem cell) persistent chez les nymphes (infectées au stade larve hexapode) et les jeunes adultes (infectés au stade nymphe) lorsque ceux-ci sont gardés à jeun. Ainsi, au laboratoire, des nymphes gardées 2 mois et des jeunes adultes gardés 4 mois conservent des sporocystes capables d'excyster.

Après la prise d'un nouveau repas sanguin sur crapaud non infecté, les sporocystes persistent dans ces cellules qui peuvent donner des cellules sessiles capables de s'hypertrophier normalement.

\section{b - Sporocystes formés dans les cellules digestives sessiles}

Les sporocystes qui se sont développés dans ces cellules sont éliminés progressivement dans les fèces au fur et à mesure que ces cellules dégénèrent.

La prise d'un nouveau repas sanguin (nymphe ou adulte) favorise l'expulsion de ces vieilles cellules et des sporocystes qu'elles libèrent.

Une partie des sporocystes peut donc persister longtemps chez le vecteur, l'autre partie étant disséminée dans le milieu extérieur.
Ces sporocystes disséminés ne résistent pas à la dessiccation; par contre dans l'eau, ils conservent pendant une dizaine de jours la possibilité d'excyster.

\section{ÉVOLUTION DE L'INFECTION CHEZ LE CRAPAUD}

Les formes parasitaires observées (fig. 2) sont :

- des gamétocytes jeunes et mûrs dans les globules rouges du sang périphérique;

- des schizontes et kystes dans les globules rouges essentiellement du foie et de la rate mais également dans les autres tissus;

- des schizontes, des kystes et des gamétocytes dans les cellules réticulo-endothéliales non pigmentées des différents organes;

- des kystes et des gamétocytes dans les cellules pigmentées du système réticulo-endothélial du foie et de la rate.

\section{1 - INFECTION NATURELLE}

\section{a - Gamétocytémie sanguine}

L'hémogrégarine est fréquente chez les crapauds de la région de Belém, $30 \%$ des animaux (120 observés) présentent des gamétocytes dans les hématies du sang périphérique lors de la capture.

Certaines de ces infections naturelles sont fortes, le pourcentage d'hématies parasitées par des gamétocytes peut atteindre $20 \%$.

Sur ces frottis effectués après la capture, la majorité des gamétocytes est mûre (fig. $2 \mathrm{H}$ ). Dans la plupart des cas il existe en même temps une faible proportion de formes jeunes (fig. $2 \mathrm{~K}$ ); chez quelques rares individus ces formes immatures peuvent être prédominantes.

La parasitémie sanguine de 4 Bufo naturellement parasités a pu être suivie pendant plusieurs mois au laboratoire; ces crapauds ont été capturés juste avant leur départ pour la France :

- Deux crapauds avaient une infection faible (Bufo $893 \mathrm{ET}$ ) ou très faible (Bufo $876 \mathrm{ET}$ ), surtout constituée

Fig. 1. - $H$. stellata chez $A$. rotondatum; $\mathrm{A}$ à $\mathrm{F}$, en coupes histologiques, $\mathrm{G}$ à $\mathrm{L}$, en frottis par appositions.

A : Division du noyau du microgamétocyte en deux après la conjugaison, $\times 1000$. B : Accumulation de parasites à gauche et jeune oocyste sphérique à un noyau, à droite, dans les cellules digestives sessiles, $\times 1000 . \mathrm{C}:$ Pénétration des sporokinètes (flèche) dans les cellules digestives souches et début de division; partie basale de l'épithélium digestif (be), $\times 1$ 000. D : Phagocytose des hématies et des gamétocytes encore dans leur coque, $24 \mathrm{~h}$ après la fin du repas infectant; cellules phagocytaire (Cp), cellule sessile (Cs), lumière cœcas digestifs $(\mathrm{Lc}), \times 500$. E : Fécondation, en haut, avec un pronucléus mâle $(\mathrm{m})$ et un pronucléus femelle (f) dans le noyau du macrogamétocyte; microgamète résiduel $(\mathrm{r}), \times 1000 \mathrm{~F}:$ Division de l'oocyste, les noyaux se répartissent le long des parois de l'étoile, $\times 500$.

G : Microgamète (flèches), $\times 1000 . \mathrm{H}:$ Oocyste en étoile, stade 1 noyau $(\mathrm{n}), \times 500$. I : Sporozoïte; complexe apical (ca), noyau (n) et cristalloïde $(\mathrm{cr}), \times 1000 . \mathrm{J}:$ Microgamétocyte après la division du noyau $(\mathrm{n}), \times 1000 . \mathrm{K}:$ Appariement d'un macrogamétocytẻ (en forme de croissant) et d'un microgamétocyte (rond), $\times 1000$. L : Sporokinètes; cristalloïde (cr), noyau (n), $\times 1000$. 

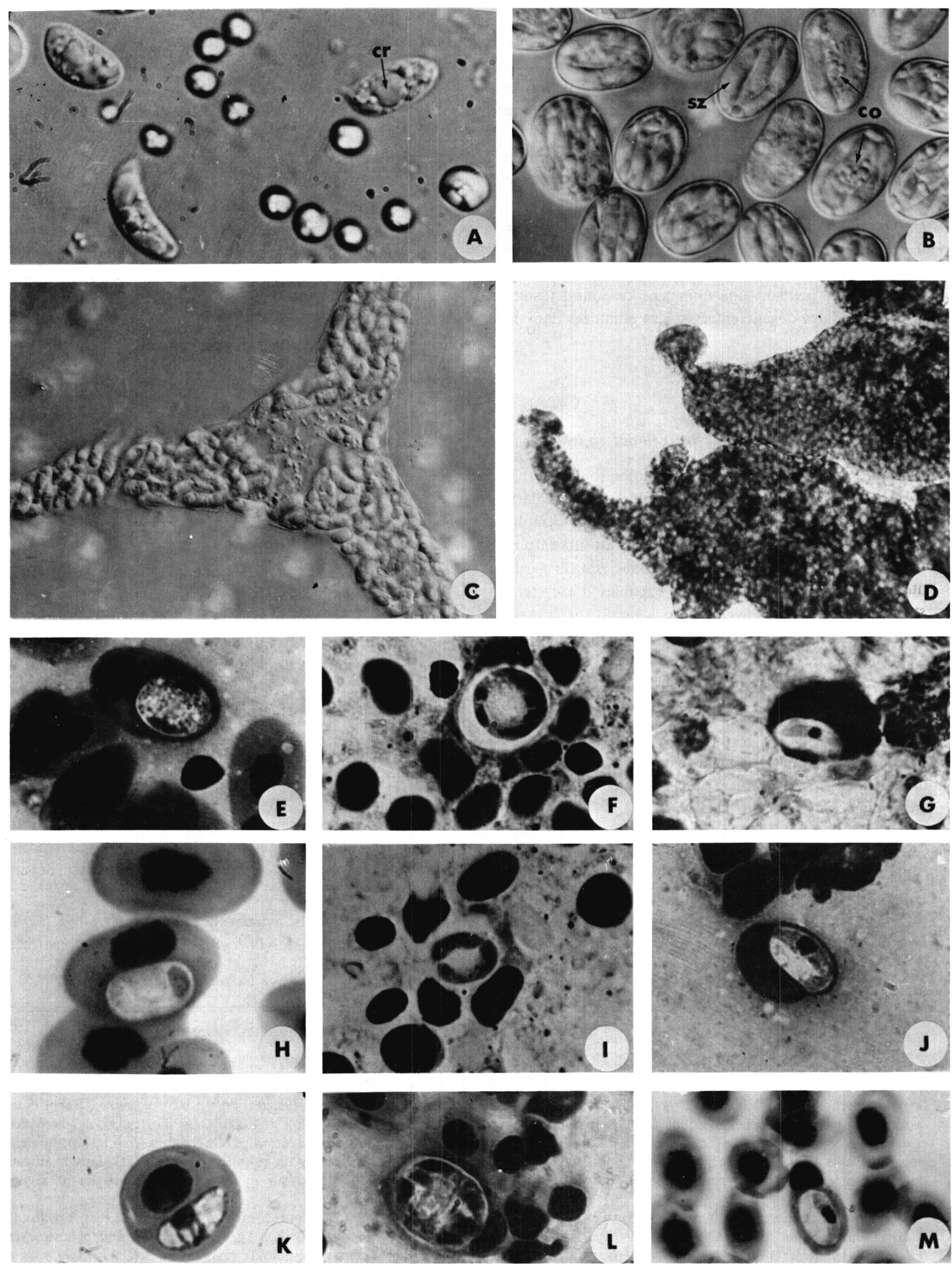
de gamétocytes mûrs, qui n'a pas variée pendant respectivement 5 et 6 mois avant autopsie.

- Un crapaud avait une infection très forte lors de la capture (Bufo $889 \mathrm{ET}$ ) avec $20 \%$ d'hématies parasitées et qui s'est maintenue à ce niveau pendant 1 an avant l'autopsie.

- Le dernier crapaud avait une infection forte lors de la capture (Bufo $877 \mathrm{ET}$ ) avait $8 \%$ d'hématies parasitées, elle a ensuite diminué régulièrement jusqu'au $3^{\mathrm{e}}$ mois $(0,5 \%$ d'hématies parasitées) avant de connaître une forte recrudescence au $5^{\mathrm{e}}$ mois peu avant l'autopsie.

\section{$\mathrm{b}$ - Formes de multiplication et kystes}

Qu'ils soient intra- ou exo-érythrocytaires, les schizontes et les kystes sont essentiellement rencontrés dans les viscères après autopsie. La proportion relative des différents stades parasitaires est variable selon l'organe et le crapaud; elle a pu être étudiée chez deux Bufo (49 ET et 877 ET). Les observations sont résumées dans le tableau I; nous faisons plusieurs remarques:

- chez le Bufo 877 ET qui est en pleine phase de recrudescence de la gamétocytémie, la shizogonie est surtout exoérythrocytaire, mais une partie se produit également dans les hématies. Les kystes forment des accumulations importantes dans les cellules pigmentées et non pigmentées du système réticulo-endothélial;

- chez le Bufo $49 \mathrm{ET}$, autopsié juste après sa capture, les stades parasitaires (schizontes, kystes et gamétocytes) sont presque uniquement intra-érythrocytaires. Nous n'observons aucune accumulation de parasites dans le système réticulo-endothélial.

\section{2 - INFECTION EXPÉRIMENTALE}

a - Résultats des expériences de transmission (tableau I)

La transmission expérimentale du parasite a été obtenue chez huit crapauds sur onze :

- les deux B. marinus de Belém (868 ET et 909 ET) qui ont ingéré des tiques à sporocystes;

- les deux B. marinus de Guadeloupe qui ont ingéré des tiques à sporocystes (1007 ET et $1008 \mathrm{ET})$;
- les trois B. poepigii du Pérou (38, 39 et 40 ES) sur les quatre qui ont ingéré des sporocystes;

- le B. poepigii 588 XP qui a ingéré du foie contenant des kystes.

Les deux B. marinus de Guadeloupe qui ont ingéré seulement des tiques à sporokinètes n'ont pas développé de parasitémie.

\section{b - Gamétocytémie sanguine}

Dans toutes les infections expérimentales développant une gamétocytémie, les premiers gamétocytes apparaissent dans le sang du doigt entre 40 et 45 jours après l'infestation.

Les deux B. marinus de Belém n'ont pas présenté le même type de gamétocytémie : le Bufo 868 ET a eu une parasitémie élevée (10\% d'hématies parasitées) et durable ( 8 mois) avant l'autopsie; le Bufo $909 \mathrm{ET}$ a eu un fort et bref pic de gamétocytémie (15\% d'hématies parasitées au $2^{\mathrm{e}}-3^{\mathrm{e}}$ mois); l'infection sanguine est ensuite devenue brutalement faible et résiduelle jusqu'au $8^{\mathrm{e}}$ mois, date de l'autopsie.

— Les B. poepigii ont eu des gamétocytémies très faibles.

\section{c - Formes de multiplication et kystes}

L'analyse des autopsies est résumée dans le tableau I. Nous remarquons que la schizogonie est surtout intraérythrocytaire dans ces infections expérimentales.

\section{DESCRIPTION MORPHOLOGIQUE}

\section{1 - Stades chez le CRapaud Gamétocytes}

a - Gamétocytes intra-érythrocytaires (fig. $2 \mathrm{H}$ et $2 \mathrm{~K}$ )

Les gamétocytes sont situés dans des hématies dont le noyau est parfois déplacé par le parasite, mais qui ne sont en général ni déformées ni modifiées. Le polyparasitisme par deux ou trois gamétocytes dans le même globule rouge peut être observé dans les fortes parasitémies.

Les jeunes gamétocytes n'ont pas de coque. Ils sont allongés, relativement trapus, avec une extrémité arrondie,

Fig. 2. - H. stellata chez A. rotondatum, en observations à frais (A à D); $H$. stellata chez B. marinus, en appositions (E à M). A : Sporokinètes libres avec un cristallö̈de réfringent $(\mathrm{cr}), \times 1000$. B : Sporocystes mûrs dans lesquels on distingue les sporozoïtes (sz) et le corps résiduel (co), $\times 1000 . \mathrm{C}$ : Oocyste étoilé mûr avec sporokinètes $(\mathrm{sp}), \times 500 . \mathrm{D}:$ Cœcas digestifs complètement envahis par les sporocystes (taches claires), $\times 50$. E : Jeune schizonte intra-érythrocytaire à deux noyaux, $\times 1000$. F : Schizonte mûr dans une cellule non pigmentée du système réticulo-endothélial, $\times 1000$. G : Kyste à un cystozoïte dans une cellule pigmentée du système réticulo-endothélial, $\times 1000$. H : Gamétocyte mûr, $\times 1200$. I : Schizonte à deux mérozoïtes dans une cellule non pigmentée du système réticulo-endothélial, $\times 1000$. J : Kyste à un cystozoïte dans une cellule non pigmentée du système réticulo-endothélial, $\times 1000 . \mathrm{K}$ : Jeune gamétocyte, $\times 1200$. L : Schizonte encore immature intra-érythrocytaire, $\times 1000$. M : Kyste intra-érythrocytaire à un cystozoïte, $\times 1000$. 


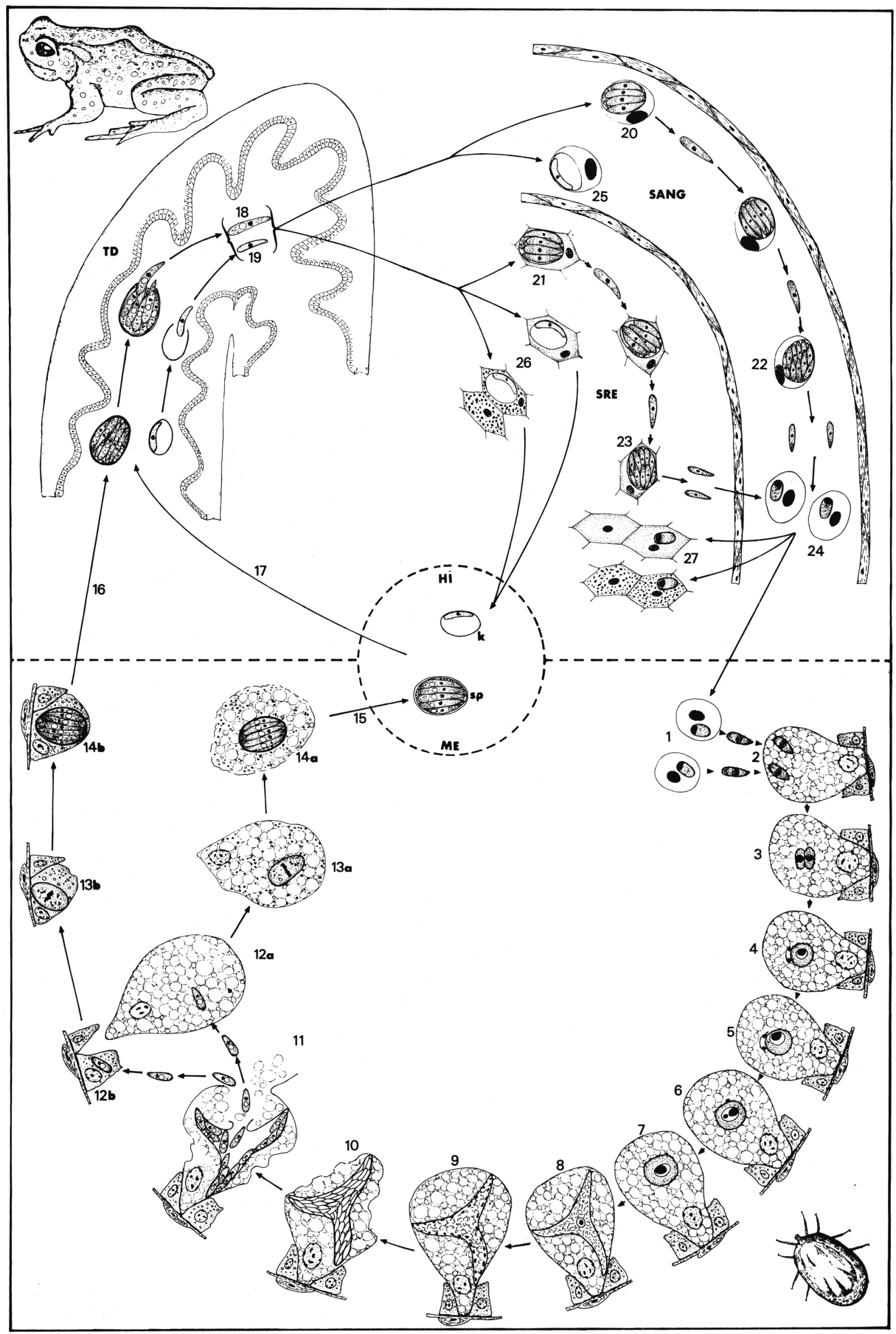

Fig. 3. - Schéma du cycle d'H. stellata (cf. discussion, paragraphe 1). 
l'autre pointue. La chromatine nucléaire est condensée sous forme de rubans transversaux. Le cytoplasme clair contient de petites vacuoles et quelques grains azurophiles.

Les gamétocytes mûrs présentent une forme en " gélule »; ils sont entourés par une coque épaisse peu perméable qui les rend peu chromophiles. Le noyau est situé à une des extrémités, le cytoplasme est clair avec de petites vacuoles. Des gamétocytes non colorés, dont seule la coque est visible, sont fréquents. Aucun dimorphisme sexuel n'a été observé. Dimensions du gamétocyte à maturité : $9 \pm 0,36 \mu \mathrm{m}$ $\times 5,13 \pm 0,75 \mu \mathrm{m}$ (frottis, Giemsa).

\section{b - Gamétocytes tissulaires exo-érythrocytaires}

Les gamétocytes peuvent former des accumulations importantes dans les cellules réticulo-endothéliales pigmentées. Une partie de ces gamétocytes a un aspect normal, mûr; les autres sont en état de lyse plus ou moins avancée.

\section{Schizontes}

\section{a - Schizontes intra-érythrocytaires (fig. 2 E et 2 L)}

Les schizontes sont ovalaires, parfois presque sphériques, situés principalement dans les globules rouges du foie, de la rate et du poumon plus rarement au niveau du cœur ou du sang périphérique.

L'hématie est hypertrophiée, son noyau est aplati, repoussé vers la périphérie et à maturité, il ne reste, surtout avec les plus gros schizontes, qu'une mince bande cytoplasmique difficile à identifier. Le schizonte est entouré d'une enveloppe assez épaisse.

Le nombre de mérozoïte varie entre 2 et 24 mérozoïtes. Les schizontes à faible nombre de mérozoïtes $(2,4,6)$ contiennent des éléments de grande taille. Les schizontes à grand nombre de mérozoïtes contiennent des éléments de petite taille. Pour des schizontes à nombre de mérozoïtes intermédiaire, la taille des mérozoïtes est variable entre les deux extrêmes précédents.

Taille des schizontes mûrs et nombre de mérozoïtes : $13,5 \mu \mathrm{m} \times 11 \mu \mathrm{m}(n=2), 13,5 \mu \mathrm{m} \times 11,5 \mu \mathrm{m}(n=4)$, $14 \mu \mathrm{m} \times 12 \mu \mathrm{m}(n=4), 15 \mu \mathrm{m} \times 15 \mu \mathrm{m}(n=4)$, $16 \mu \mathrm{m} \times 13 \mu \mathrm{m}(n=12), 16 \mu \mathrm{m} \times 14 \mu \mathrm{m}(n=14)$, $16 \mu \mathrm{m} \times 15 \mu \mathrm{m}(n=16), 15 \mu \mathrm{m} \times 14,5 \mu \mathrm{m}(n=24)$ (frottis, Giemsa).

En coupe histologique, les schizontes mûrs paraissent obstruer les capillaires sanguins et il est fréquent d'observer une accumulation d'hématies en amont du parasite.

\section{b - Schizontes tissulaires exo-érythrocytaires}

\section{(fig. 2 I et 2 F)}

Ils sont observés uniquement dans les cellules non pigmentées du système réticulo-endothélial. Ils ont une morphologie peu différente des schizontes intra-érythrocytaires, mis à part une taille un peu plus petite et qui ne varie pratiquement pas. Le nombre de mérozoïtes par schizonte varie entre 2 et 14 .

Taille des schizontes mûrs et nombre de mérozoïtes : $9 \times 7 \mu \mathrm{m}(n=2), 11 \times 8 \mu \mathrm{m}(n=2), 11 \times 7 \mu \mathrm{m}$ $(n=2), 11 \times 8 \mu \mathrm{m}(n=2), 9 \times 7 \mu \mathrm{m}(n=2)$, $12 \times 10 \mu \mathrm{m}(n=2), 11 \times 9 \mu \mathrm{m}(n=2), 10 \times 9 \mu \mathrm{m}$ $(n=3), 13 \times 10 \mu \mathrm{m}(n=3), 10 \times 8 \mu \mathrm{m}(n=4)$, $12 \times 10 \mu \mathrm{m}(n=5), 13 \times 10 \mu \mathrm{m}(n=8), 11 \times 9 \mu \mathrm{m}$ $(n=8), 11 \times 9 \mu \mathrm{m}(n=10)$ (frottis, Giemsa).

\section{KYSTES}

\section{a - Kystes intra-érythrocytaires (fig. $2 \mathrm{M}$ )}

Les kystes sont observés comme les schizontes, le plus souvent au niveau du foie et de la rate. Dans de rares cas nous les observons dans les hématies du sang périphérique.

Ils ont une forme ovalaire, et sont entourés d'une enveloppe épaisse. Ils renferment un cystozoïte unique, allongé sur le bord d'une masse cytoplasmique résiduelle peu colorable.

Le cystozoïte est un vermicule allongé dont une extrémité est repliée; le noyau petit et dense est plus proche de l'autre extrémité.

Le kyste mesure en moyenne $11,8 \pm 0,5 \mu \mathrm{m} \times 6,6$ $\pm 0,4 \mu \mathrm{m}$, le cystozoïte $12,7 \pm 0,5 \mu \mathrm{m} \times 2,6 \pm 0,5 \mu \mathrm{m}$ (frottis, Giemsa).

\section{b - Kystes exo-érythrocytaires (fig. $2 G$ et $2 \mathrm{~J}$ )}

Les kystes sont observés dans les cellules pigmentées et non pigmentées du système réticulo-endothélial ou ils peuvent s'accumuler sans montrer de signe de dégénérescence. Nous observons des kystes à un cystozoïte de même morphologie que dans les hématies et beaucoup plus rarement des kystes à deux cystozoïtes.

\section{2 - Stades chez la tique Gamétocyte mobile}

Le gamétocyte qui sort de sa coque dans la lumière des coecas digestifs de la tique est court, avec une extrémité effilée. Le noyau est central, de grande taille et à chromatine très dense.

Dimensions du gamétocyte : 6,6 $\pm 0,5 \mu \mathrm{m} \times 2,1$ $\pm 0,2 \mu \mathrm{m}$ (apposition, Giemsa).

Nous n'observons pas de dimorphisme sexuel à ce stade.

\section{CONJUGAISON-FÉCONDATION}

Après pénétration dans les cellules digestives les gamétocytes deviennent rapidement sphériques. Ils peuvent être isolés mais forment le plus souvent des groupements serrés dans une même cellule (fig. 1 B). Nous n'avons pas pu observer les stades précoces d'appariement de deux gamétocytes, par contre les stades plus évolués sont nombreux : 
- sur apposition le macrogamétocyte forme alors un croissant qui entoure complètement le microgamétocyte sphérique (fig. $1 \mathrm{~K}$ ). Le noyau du microgamétocyte a un aspect homogène, il est sphérique dans un cytoplasme clair à petites vacuoles, le noyau du macrogamétocyte, un peu plus dense, est étiré dans un cytoplasme basophile à petites vacuoles claires;

- sur coupe cet aspect " enchâssé " n'est pas retrouvé, le macrogamétocyte est simplement accolé au microgamétocyte et son noyau est sphérique.

La fécondation a été observée uniquement en coupe et suit un schéma classique.

- Avant la fécondation proprement dite, le noyau du macrogamète est formé d'une masse sphérique très dense (pronucléus femelle) dans un nucléoplasme clair. En même temps, le noyau du microgamétocyte s'est divisé en deux parties et son cytoplasme est devenu très vacuolaire (fig. $1 \mathrm{~A}$ et $1 \mathrm{~J})$.

- Deux microgamètes se forment, ils ont le corps enflé et un flagelle court et épais (fig. $1 \mathrm{G}$ ). (Longueur du corps $=4 \mu \mathrm{m}$, longueur du flagelle $=4 \mu \mathrm{m}$ ).

Un des deux microgamètes pénètre le noyau du macrogamète et il est fréquent d'observer le pronucléus mâle (plus petit) à côté du pronucléus femelle avant que ne se forme l'oocyste.

\section{OOCYSTE}

Le jeune oocyste ne se distingue du macrogamète que par la présence du résidu du microgamète non fécondant qui reste accolé à l'oocyste.

Le polyparasitisme est fréquent dans une même cellule digestive au début du développement mais nous n'observons par la suite qu'un seul oocyste mûr par cellule.

L'oocyste à d'abord une forme sphérique (diamètre de $20 \mu \mathrm{m}$ en coupe), il prend ensuite une forme triangulaire puis étoilée (fig. $1 \mathrm{H}$ ). L'étoile est généralement constituée de 3 à 6 branches, dans certains cas, seulement 2 branches sont formées. La taille définitive de l'étoile est obtenue avant que l'oocyste n'ait commencé à se diviser.

- En apposition, le cytoplasme est basophile parsemé de nombreuses petites vacuoles qui lui donnent un aspect spongieux classique chez les oocystes jeunes de Coccidies; le noyau de grande taille est situé dans une des branches.

- En coupe, les bras sont parfois repliés à l'intérieur de la cellule hôte.

La phase de division suit cette phase de croissance, les noyaux issus de nombreuses divisions se placent régulièrement le long des parois de l'étoile ( $(f i g .1 \mathrm{~F})$. Elle aboutit à la formation de sporokinètes (selon la terminologie de Reichenow, 1920) dont le nombre est de l'ordre de 100 à 150 (fig. $2 A$ et $2 C$ ). Ces sporokinètes s'individualisent par bourgeonnement en périphérie de l'oocyste; au centre, il subsiste un corps résiduel qui a la forme de l'étoile.

A maturité de l'étoile, en observation à frais ou sur apposition, une branche mesure entre $90 \mu \mathrm{m}$ et $120 \mu \mathrm{m}$ de long.

Les cellules digestives dans lesquelles s'effectue le développement de l'oocyste sont rapidement modifiées : le cytoplasme est plus basophile et la chromatine nucléaire est plus dispersée que dans les cellules saines. Vers le $10^{\mathrm{e}}$ jour, la cellule parasitée est réduite à une mince bande cytoplasmique et à un noyau picnotique. Après, la cellule hôte n'est plus visible.

\section{SPOROKINÈTES (fig. 1 L et 2 A)}

Le sporokinète libre mesure 14,7 $\pm 0,6 \mu \mathrm{m}$ de long sur $4,3 \pm 0,3 \mu \mathrm{m}$ de large (à frais).

Il est animé de mouvements ondulatoires, la partie arrière est plus mince.

Le cristalloïde occupe une place importante, le plus souvent sous forme de deux masses entre lesquelles le noyau dense est aplati; il peut également se présenter en une seule masse.

\section{SPOROBLASTES ET SPOROCYSTES (fig. 2 B)}

Les sporokinètes qui ont pénétré dans de nouvelles cellules digestives subissent alors une phase de croissance pour atteindre la taille et la forme définitive du sporocyste; lors de cette phase, le cristalloïde se morcelle. Le noyau du sporokinète se divise ensuite; le cytoplasme est alors basophile, vacuolaire et le cristalloïde n'est plus visible. Les sporozoïtes se différencient ensuite en même temps que se forme l'enveloppe résistante du sporocyste.

De forme ovale, le sporocyste mesure à frais 19,5 $\pm 0,63 \mu \mathrm{m}$ de long sur $14,19 \pm 0,61 \mu \mathrm{m}$ de large. Il contient les sporozoïtes et un corps résiduel le plus souvent central et sphérique $(\varnothing=4 \mu \mathrm{m})$ ou parfois morcelé. Ce corps résiduel est constitué de petits granules réfringents.

Écrasés entre lame et lamelle, les sporozoïtes sortent avec des mouvements lents par une déchirure à une des extrémités du sporocyste. Lorsqu'on les fait excyster dans une solution de trypsine, ils s'ouvrent complètement en deux parties égales, suivant l'axe longitudinal; il est alors possible de compter le nombre de sporozoïtes par sporocyste; il varie entre 8 et 24 pour une moyenne de 16 .

\section{SPOROzOÏTE (fig. 1 I)}

Il est vermiforme et mesure à frais 17,6 $\pm 0,82 \mu \mathrm{m}$ de long sur 3,3 $\pm 0,39 \mu \mathrm{m}$ de large.

Le noyau est central, à chromatine condensée, situé à proximité d'un cristalloïde unique. Le cytoplasme est granulaire, sauf à une extrémité ou il existe une structure d'aspect plus fibrillaire correspondant au complexe apical.

Il est moins mobile que le sporokinète. 


\section{PARASITES ABORTIFS}

Nous distinguons :

- des gamétocytes dans les cellules digestives phagocytaires; certains ont un aspect normal, d'autres sont en voie de lyse;

- des macrogamétocytes non fécondés, dans les cellules digestives sessiles et phagocytaires; ils sont hypertrophiés mais n'évoluent plus;

- des oocystes dans les cellules sessiles (oocyste sphérique à un noyau, oocyste en étoile à un noyau, oocyste plurinucléé).

Tous ces parasites seront éliminés dans les fèces après le $15^{\mathrm{e}}$ jour en même temps que la cellule hôte.

\section{DISCUSSION}

\section{1 - SChÉma du CyCle (fig. 3)}

Les gamétocytes mûrs (1) dans l'hématie du crapaud sont ingérés par la tique; ils sortent de leur coque dans les cœcas digestifs et pénètrent dans les cellules digestives sessiles (2). Certains gamétocytes encore dans leur coque sont ingérés par les cellules digestives phagocytaires et n'évoluent plus. Un macrogamétocyte et un microgamétocyte s'apparient (3) selon le mode adeleidéen, puis le noyau du microgamétocyte se divise en deux (4) et deux microgamètes sont formés (5). Le noyau d'un des deux microgamètes pénètre dans le noyau du macrogamète (6). La fécondation donne naissance à un oocyste sphérique (7), contre lequel reste appliqué le microgamète résiduel. L'oocyste grossit en prenant une forme en étoile à un seul noyau (8), il se divise ensuite (9) pour former l'oocyste mûr contenant 100 à 150 sporokinètes (10). L'oocyste se rompt (11) et libère les sporokinètes qui entrent dans de nouvelles cellules digestives (12). Ces cellules sont soit des cellules sessiles (12 $a$ ) soit des cellules souches $(12 b)$; le sporokinète grossit (13a et $b$ ), puis se divise pour former un sporocyste qui contient entre 8 et 24 sporozoïtes (14 $a$ et $b$ ). Les sporocystes formés dans les cellules digestives sessiles sont progressivement éliminés dans la lumière du tube digestif puis dans les fèces et le milieu extérieur (15); les sporocystes formés dans les cellules souches persistent chez la tique. Le crapaud s'infecte (cf. transmission (fig. 4)) en ingérant : - soit directement la tique (16), - soit les sporocystes (sp) transitant dans le milieu extérieur (ME) où chez un hôte intermédiaire (HI) (17), - soit les kystes (k) transitant dans le milieu extérieur où chez un hôte intermédiaire (17).

Les sporozoïtes (18) ou les cystozoïtes (19) sont libérés dans le tube digestif (TD); ils donnent naissance soit à la schizogonie (20-22 et 21-23) soit à des kystes (25-26), à la fois dans les hématies et dans le système réticuloendothélial (SRE). Les schizontes sont d'abord à grands mérozoïtes peu nombreux (20-21) puis apparaissent des schi-

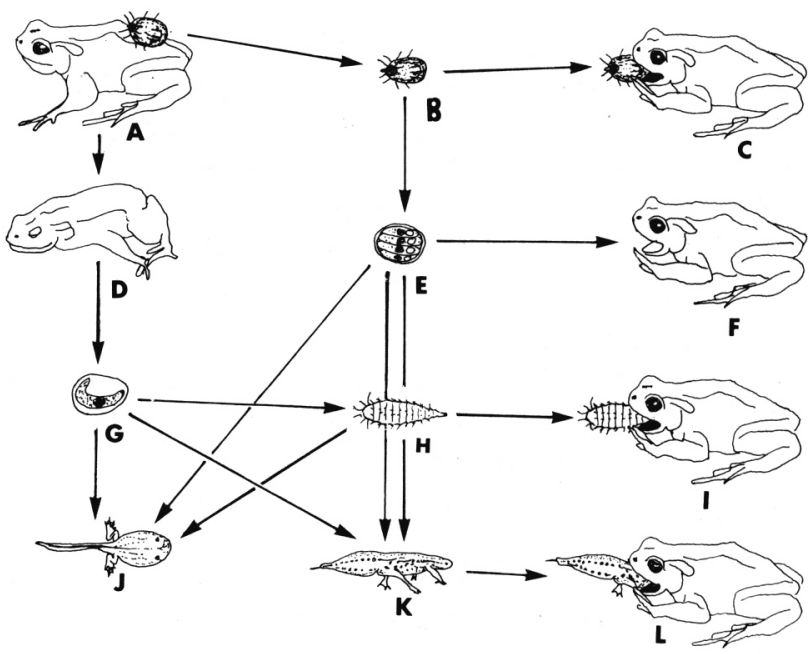

Fig. 4. - Hypothèses sur la transmission naturelle d'H. stellata ( $c f$. discussion, paragraphe 3 ).

zontes à nombreux mérozoïtes de petites tailles (22-23) qui donnent naissance aux gamétocytes. Les kystes peuvent s'accumuler dans les cellules du système réticulo-endothélial où ils persistent et constituent des formes d'attente.

Dans les infections anciennes, les gamétocytes sont phagocytés dans les cellules pigmentées et non pigmentées (27) du système réticulo-endothélial où ils sont détruits.

\section{2 - InTERPRÉTATION DU CYCLE}

\section{a - Chez la tique}

L'abondance du matériel nous a permis de suivre aisément la succession chronologique des différents stades parasitaires. Seul l'accolement des gamétocytes mâle et femelle au début de la conjugaison n'a pas été observé.

\section{b - Chez le crapaud}

\section{- Kystes :}

Les kystes ont été observés dans le système réticuloendothélial comme chez Hepatozoon domerguei (Landau et coll., 1972), mais également dans une localisation inhabituelle, le globule rouge. Comme dans le genre Hepatozoon, ces kystes se sont révélés expérimentalement infectants pour des crapauds sains dans lesquels ils déterminent à la fois la schizogonie et la formation d'autres kystes.

Nous n'avons pas pu suivre la formation des kystes à partir du sporozoïte, mais il parait logique de penser qu'elle se calque sur celle observée avec Hepatozoon.

Dans notre schéma, nous les faisons dériver directement soit du sporozoïte lorsque l'infestation a lieu avec des sporocystes soit d'un cystozoïte, lorsque l'infestation se fait par prédation. 


\section{- La schizogonie}

Nous retrouvons les mêmes deux types de schizontes à grands mérozoïtes et à petits mérozoïtes que chez Hepatozoon (Mackerras, 1962; Ball et coll., 1969; Landau et coll., 1972) et que dans le genre Karyolysus (Reichenow, 1920).

Dans les infections expérimentales étudiées ici, les autopsies ont été réalisées tardivement alors que les deux types de schizontes étaient présents et que les gamétocytes avaient envahi les hématies. Nous avons donc procédé par analogie avec les hémogrégarines précédentes et placés dans le schéma les schizontes à petits et nombreux mérozoïtes à l'origine des gamétocytes.

\section{- Accumulation des gamétocytes \\ dans le système réticulo-endothélial}

Les gamétocytes, particulièrement dans certaines infections anciennes, forment des accumulations dans les cellules pigmentées et non pigmentées du système réticulo-endothélial. L'observation de formes lysées indique que leur destruction se fait dans cette localisation. Il s'agit là d'un phénomène différent de celui d'H. prodhoni (Landau, 1973) où les gamétocytes sont stockés mais non détruits dans le système réticuloendothélial qui constituerait une réserve de gamétocytes. Ces gamétocytes seraient libérés à la mort de la cellule de stockage et repris ensuite par les monocytes du sang périphérique.

\section{3 - TRANSMISSION}

Dans le cycle expérimental que nous avons figuré, le crapaud s'infecte directement en mangeant la tique mais nous ignorons si, dans la nature, il s'agit du mode d'infestation régulier du crapaud.

Théoriquement, tous les mécanismes de transmission connus chez les Coccidies peuvent être utilisés : - excrétion de stades infectants dans le milieu extérieur (sporocystes); sporogonie chez un vecteur hématophage; - prédation et ingestion de kystes d'attente tissulaires.

Si l'on tient compte du caractère omnivore de $B$. marinus plusieurs modes de transmission paraissent possibles (fig. 4).

\section{$\mathrm{a}-$ Alimentation de $\mathrm{B}$. marinus}

En Guadeloupe, Lescure (1979), observe que B. marinus adulte ingère de nombreux insectes, quelques gastéropodes et parfois des crabes et des batraciens. En fait, ils peuvent ingérer toute proie en mouvement arrivant à proximité, même s'il s'agit d'une feuille tombant d'un arbre ou d'un leurre (Alexander, 1964) ; ce dernier auteur constate également que ces Bufo peuvent ingérer des aliments inertes (viandes ou végétaux).

Nous avons constaté d'autre part au laboratoire qu'ils pouvaient ingérer spontanément des tiques gorgées et des souris blanches vivantes (cf. matériels et méthodes).

L'alimentation du tétard est mal connue, ils semblent très opportunistes également et capables de manger aussi bien des débris animaux et végétaux que des proies vivantes (larves d'arthropode). b - Hypothèses sur la transmission (fig. 4)

\section{Par les sporocystes}

Les sporocystes sont formés chez la tique (B) gorgée sur le Bufo parasité (A). Plusieurs voies d'infestation sont envisageables :

- le crapaud sain (C) s'infecte en ingérant la tique (B), parasitée par des sporocystes;

- le crapaud sain $(F)$ ingère des débris animaux ou végétaux contaminés par les sporocystes excrétés $(\mathrm{E})$;

- le crapaud sain (I) ingère une larve d'arthropode $(\mathrm{H})$ qui a ingéré les sporocystes excrétés;

- le crapaud sain $(\mathrm{L})$ ingère un petit vertébré $(\mathrm{K})$ qui a ingéré soit directement les sporocystes excrétés, soit une larve d'arthropode qui a ingéré ces sporocystes;

- le tétard sain $(\mathrm{J})$ ingère soit directement les sporocystes excrétés, soit une larve d'arthropode qui a ingéré ces sporocystes.

Chez Karyolysus une partie des sporocystes est également disséminée dans le milieu extérieur. Reichenow (1920) pense que le vertébré s'infecte essentiellement en ingérant l'arthropode parasité; il évoque également la possibilité d'un léchage des sporocystes libres par le lézard.

\section{Par les kystes}

Les kystes $(\mathrm{G})$ sont ingérés par un prédateur après la mort d'un crapaud parasité (D). Plusieurs voies d'infestation sont proposées :

- le crapaud sain (L) dévore un petit vertébré $(\mathrm{K})$ ou une larve d'arthropode $(\mathrm{H})$ qui ont ingéré des kystes;

- le tétard sain $(\mathrm{J})$ ingère directement les kystes ou une larve d'arthropode qui a ingéré les kystes.

Le seul autre genre d'Hémogrégarine chez lequel nous retrouvons des kystes est Hepatozoon. Le mode de transmission dépend des habitudes alimentaires de l'hôte (Landau et coll., 1973). Un lézard pourra s'infecter en mangeant des moustiques chez lesquels s'est effectuée la sporogonie. Au contraire, un serpent ne peut s'infecter qu'en dévorant une proie (un lézard en particulier, comme cela a été démontré expérimentalement) dont les tissus contiennent des kystes.

En conclusion, il est difficile de s'attacher à une seule hypothèse. Nous pensons que toutes les voies décrites sont fonctionnelles mais qu'en fonction des variations de l'environnement l'une ou l'autre peut être privilégiée.

\section{4 - Statut TAXonomiQue}

En raison des différences exposées ci-dessous, séparant notre parasite des autres hémogrégarines connues, nous le classons dans un genre nouveau et le nommons Hemolivia stellata n. g., s. sp.; l'espèce est désignée comme espèce type du genre. Le matériel type est un oocyste marqué sur un étalement du contenu d'une tique, coloré par le Giemsa et enregistré sous le $n^{\circ} \mathrm{P}$ XII 40 ; une partie du matériel paratype est déposé à l'Institut Evandro Chagas de Belém. 


\section{a - Caractères différentiels du genre Hemolivia}

Notre parasite se différencie des genres Haemogregarina dont les oocystes sont multisporés et Cyrilia dont les oocystes sont asporés; en outre le vecteur d'Hemolivia est un arthropode, celui d'Haemogregarina et Cyrilia une sangsue.

Les autres hémogrégarines Adeleidea auxquelles il doit être comparé sont toutes transmises par un arthropode hématophage.

Hemolivia se rapproche d'Hepatozoon, par la présence de kystes tissulaires mais il en diffère par de nombreux caractères qui sont essentiellement :

- schizontes et kystes uniquement exo-érythrocytaires chez Hepatozoon, extra- et intra-érythrocytaires chez Hemolivia; - maturation des sporocystes à l'intérieur de l'oocyste qui reste intact chez le vecteur d'Hepatozoon, rupture de l'oocyste, libération de sporokinètes, invasion d'une autre cellule, puis maturation des sporocystes chez celui d'Hemolivia.

Hemolivia est proche de Karyolysus par les caractères de la sporogonie qui, dans les deux genres, s'effectue en deux temps, avec libération de sporokinètes et maturation des oocystes dans de nouvelles cellules de l'hôte intermédiaire.

Il en diffère par :

- la présence de kystes d'attente chez Hemolivia, leur absence chez Karyolysus; - la schizogonie extra- et intraérythrocytaire chez le premier, uniquement exoérythrocytaire chez le second; - la maturation des sporocystes chez la tique qui a fait le repas infectant pour Hemolivia, la transmission héréditaire chez l'acarien vecteur de Karyolysus, dont les sporokinètes envahissent les œufs pour se transformer en sporocyste chez la descendance.

b - Définitions

- genre Hemolivia

Hémogrégarine Adeleidea, parasite de vertébrés à sang froid, présentant une gamétogonie érythrocytaire, une cystogonie et une schizogonie érythrocytaires et exo-érythrocytaires.

Le vecteur est une tique Amblyommidae.

Sporogonie évoluant en deux étapes : 1 - conjugaison, fécondation et formation d'un oocyste contenant des sporokinètes; 2 - libération des sporokinètes qui pénètrent chez le même individu dans d'autres cellules digestives pour former les sporocystes.

Il n'y a pas de transmission trans-ovarienne chez la tique.

- espèce type stellata :

Parasite de Bufo marinus dans la région de Belém (Brésil). Vecteur : Amblyomma rotondatum.
Étymologie : ce nouveau genre est dédiée à Olivia Petit qui a capturé le premier animal infecté. Le taxon est féminin.

\section{CONCLUSION}

Chez Hemolivia stellata, tous les mécanismes potentiels de transmission sont réunis. Outre le mode classique d'infestation par ingestion du vecteur chez lequel s'est déroulée la sporogonie, nous observons : $-a$ ) une excrétion par la tique de sporocystes infectants qui s'ils sont rejetés dans l'eau peuvent survivre plusieurs jours, $-b$ ) la formation de kystes d'attente infectants dans les tissus du Crapaud.

Remerciements. - Nous remercions P. Morel et J. Lescure pour la détermination des tiques et des batraciens. Nous remercions également P. Bastien, J. Y. Golvan et J. P. Pointier qui nous ont procurés des crapauds vivants. Nous exprimons notre gratitude au $\mathrm{D}^{\mathrm{r}}$ Alexandre $\mathrm{Da}$ Costa Linhares directeur de l'Institut Evandro Chagas qui nous a permis de travailler à Belem.

\section{RÉFÉRENCES}

Alexander T. R. : Observations on the feeding behaviour of Bufo marinus. Herpetologica, 1964, 20, 255-259.

Agbede R. I. S., Kemp D. H. : Digestion in the cattle-tick Boophilus microplus: light microscope study of the gut cells in nymphs and females. Int. J. Parasitol., 1985, 15, 147-157.

Ball G. H. : Hemogregarine life cycles. 2nd Inter. Cong. Parasit., Washington, 1970,9 p. polycop.

Lainson R. : On Cyrilia gomesi (Neiva and Pinto, 1926) gen. nov. (Haemogregarinidae) and Trypanosoma bourouli Neiva and Pinto, in the fish Synbranchus marmoratus: simultaneous transmission by the leech Haementeria lutzi. In : Canning, E. U., ed., Parasitological Topics: a Presentation volume to P. C. C. Garnham, F. R. S. on the Occasion of his 80th Birthday, Soc. Protozool. Spec. Publ. $\mathrm{N}^{\circ} 1$, Allen press, Lawrence, Kansas, 1981, p. $150-158$.

Landau I., Michel J. C., Chabaud A. G., Brygoo E. R. : Cycle biologique d'Hepatozoon domerguei, discussion sur les caractères fondamentaux des cycles de Coccidie. Zeitsch. Parasitenk., 1972, 38, 250-270.

Landau I. : Diversité des mécanismes assurant la pérennité de l'infection chez les Sporozoaires Coccidiomorphes. Mem. Museum Nat. Hist. Nat., nouv. sér., A, Zool., 1973, 77, 1-62.

Lescure J. : Étude taxonomique et éco-éthologique d'un Amphibien des petites Antilles : Leptodactylus phallax Müller, 1926 (Leptodactylidae). Bull. Museum Nat. Hist. Nat., 1979, $4^{\mathrm{e}}$ sér., $1, \mathrm{n}^{\circ} 3,757-774$.

Mackerras M. J. : The life history of a Hepatozoon (Sporozoa: Adeleidea) of varanid lizards in Australia. Austr. J. Zool., 1962, 10, 35-44.

Petit G., Landau I., Lainson R. : Une nouvelle Hémogrégarine, chez Bufo marinus et Amblyomma rotondatum, au Brésil. Third Mediterranean Conference of Parasitology, 1987, Jerusalem.

Reichenow E. : Los Hemococcidios de los Lacertidos. Trabajos del Museo Nacional de Ciencas Naturales. Madrid. Serie Zoologica, 1920, $n^{\circ} 40,149 \mathrm{p}$.

Walker A. R., Fletcher J. D. : Histology of digestion in nymphs of Rhipicephalus appendiculatus fed on rabbits and cattle naive and resistant to the tick. Int. J. Parasitol., 1987, 17, 1393-1411. 